\title{
Radiological improvement of psoriatic arthritis by biologic therapy
}

Toshiyuki Yamamoto

Department of Dermatology, Fukushima Medical University, Hikarigaoka 1, Fukushima 960-1295, Japan

Corresponding author: Prof. Toshiyuki Yamamoto, E-mail: toyamade@fmu.ac.jp

Sir,

Since the introduction of biologics, the treatment efficacy of psoriatic arthritis (PsA) has much improved. Biologics are usually considered to prevent the progression of bone and joint deformity, but not to recover existing deformities. Herein, a case of PsA in which a radiological improvement in the peripheral joint was achieved by biologics is reported.

A 40-year-old female visited our department complaining of skin lesions on the trunk and extremities seen for more than 8 years. Joint pain appeared one year before. Upon physical examination, scaly erythematous plaques were found coalesced, involving large areas of the trunk and extremities, with a Psoriasis Area and Severity Index (PASI) score of 24.6. Furthermore, some of the fingers were deformed and did not yield to bending (Fig. la). A biopsy specimen from the trunk revealed hyperkeratosis with parakeratosis, a regular elongation of the epidermis, and mononuclear cell infiltration around the dilated blood vessels in the papillary layer, which are the characteristic features of psoriasis. A roentgenogram revealed a narrowing of the proximal interphalangeal (PIP) joint space of the fourth and fifth right fingers (Fig. lb) and the fifth left finger. She was initially treated with infliximab $(5 \mathrm{mg} / \mathrm{kg}$ intravenously at weeks 0,2 , and 4 , followed by every 8 th week), which dramatically improved the skin lesions. She no longer complained of joint pain, as the joint pain was also reduced. However, because secondary failure for the skin lesions appeared 1 year and 2 months after the initiation of infliximab, treatment was switched to secukinumab (300 mg subcutaneously at weeks $0,1,2$, 3 , and 4 , and every 4 weeks thereafter). The cutaneous psoriasis was successfully treated with secukinumab and resulted in a clear PASI score 3 months later. Arthralgia did not relapse and remained well controlled. The crooked fingers straightened almost completely (Fig. 1c). An X-ray 1 year and 2 months after the initiation of secukinumab showed improvement in the joint space narrowing (Fig. ld).

Our case showed a radiographic improvement of PsA after treatment with biologics, which are often thought to inhibit, but not reverse, the progression of joint deformity. In this case, however, not only clinical but also radiographic remission was achieved by biologics. To date, few cases of radiological improvement of rheumatoid arthritis and juvenile idiopathic arthritis by biologics have been reported [1-4], where tocilizumab, an interleukin-6 receptor (IL-6R) antibody, restored damaged cartilages. However, as far as we know, there have been no reports showing the radiological improvement of PsA by biologics. In our case, bone erosions were repaired by anti-tumor necrosis factor (TNF) antibody and anti-IL-17A antibody, both over a 14-month treatment period. Unfortunately, no radiological evaluation was performed at the time of switching from infliximab to secukinumab. Matrix metalloproteinase (MMP) is a tissue-destroying enzyme regulated by IL-1 and TNF. In addition, Th17 plays an important role in upregulating MMP expression [5]. In our case, the joint pain was in remission, and an X-ray was done 3 years after the initiation of biologics. Hence, the limitation of our analysis is that we do not know whether the radiographic improvement was effected by infliximab or secukinumab, because at the time of the switching of the biologic, an X-ray examination was not performed. However, modulating the TNF- and Th17pathways may have altered MMP activity, reduced tissue destruction, and, therefore, improved the outcome. 

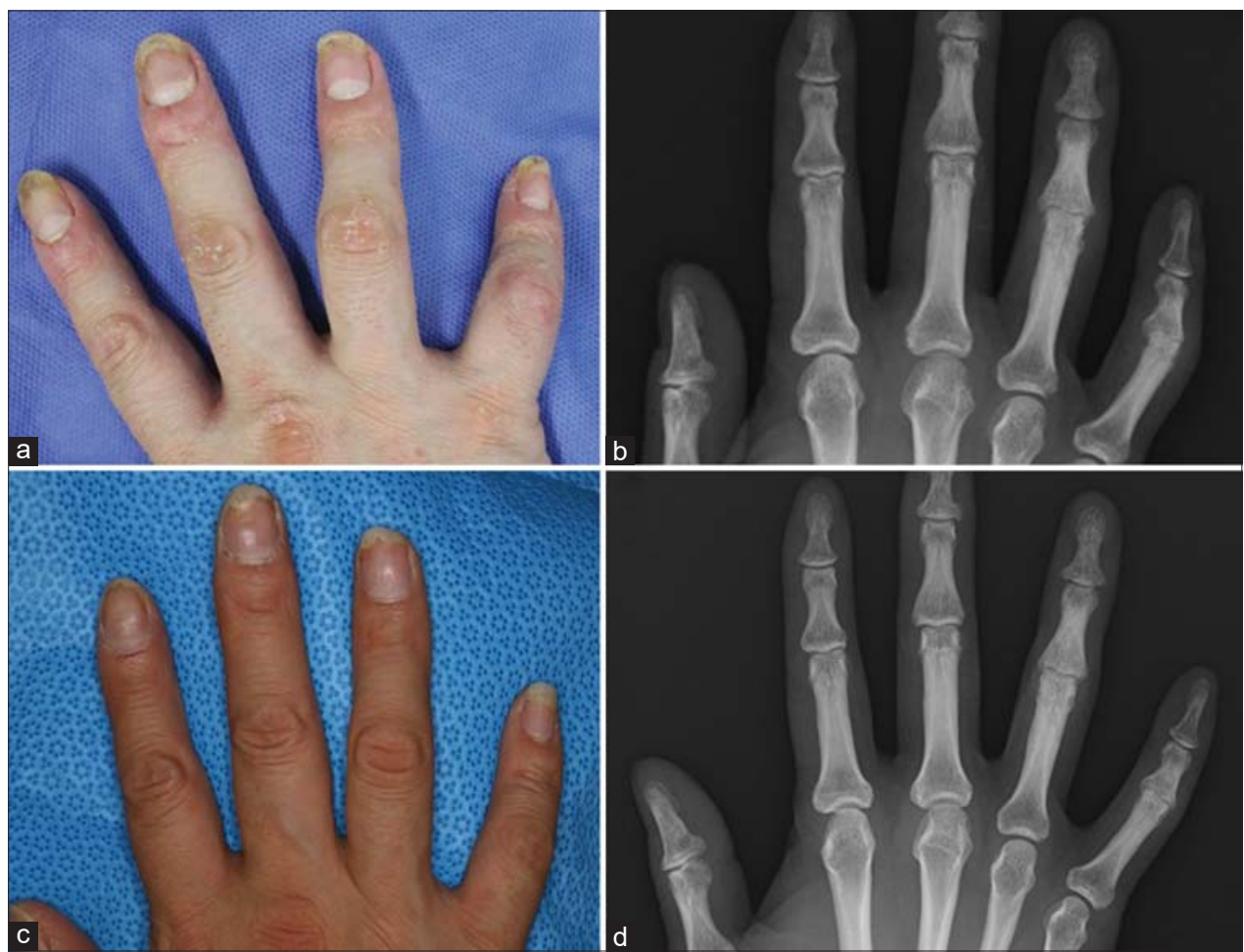

Figure 1: (a) Deformed fourth and fifth right fingers before biologic therapy. (b) Radiographic findings showing a narrowing of the PIP joint spaces of the fourth and fifth fingers. (c) Finger deformity much improved 28 months later. (d) Radiographic findings showing partial recovery of joint spaces.

\section{Consent}

The examination of the patient was conducted according to the principles of the Declaration of Helsinki.

The authors certify that they have obtained all appropriate patient consent forms, in which the patients have given consent for images and other clinical information to be included in the journal. The patients understand that their names and initials will not be published and due effort will be made to conceal their identity, but that anonymity cannot be guaranteed.

\section{REFERENCES}

1. Ramanan AV, Dick AD, Guly C, McKay A, Jones AP, Hardwick B, et al. Tocilizumab in patients with anti-TNF refractory juvenile idiopathic arthritis-associated uveitis (APTITUDE): a multicentre, single-arm, phase 2 trial. Lancet Rheumatol. 2020;2:e135-41.

2. Finzel S, Rech J, Schmidt S, Engelke K, Englbrecht M, Schett G.
Interleukin-6 receptor blockade induces limited repair of bone erosions in rheumatoid arthritis: a micro CT study. Ann Rheum Dis. 2013;72:396-400.

3. Watanabe R, Hirabayashi Y, Okuno H, Ishii T, Harigae H. Dramatic radiographic repair by tocilizumab in a very elderly patient with rheumatoid arthritis. Mod Rheumatol. 2014;24:1028-9.

4. Tozawa Y, Fujita S, Abe S, Kitamura K, Kobayashi I. Radiological improvement by tocilizumab in polyarticular juvenile idiopathic arthritis. Pediatr Int. 2015;57:307-10.

5. van Hamburg JP, Asmawidjaja PS, Davelaar N, Mus AM, Colin EM, Hazes JM, et al. Th17 cells, but not Th1 cells, from patients with early rheumatoid arthritis are potent inducers of matrix metalloproteinases and proinflammatory cytokines upon synovial fibroblast interaction, including autocrine interleukin-17A production. Arthritis Rheum. 2011;63:73-83.

Copyright by Toshiyuki Yamamoto. This is an open access article distributed under the terms of the Creative Commons Attribution License, which permits unrestricted use, distribution, and reproduction in any medium, provided the original author and source are credited.

Source of Support: Nil, Conflict of Interest: None declared. 